\title{
De la Virtualización a la Interactividad
}

\author{
(From virtualization to interactivity)
}

JAVIER CALLEJO

(UNED, España)

RESUMEN: La educación a distancia es socialmente percibida como un tipo de educación sin interactividad o, como hace la teoría, con una interactividad con particulares características. A causa de esto, el término interactividad se ha convertido en uno de los elementos centrales de la educación a distancia que, a la vez, tiene múltiples significados. Además, con el creciente uso de Internet, la propia interactividad adquiere nuevas formas. La red facilita especialmente la comunicación horizontal, entre iguales, y el uso de la imagen en los encuentros entre quienes aprenden y maestros o tutores, o sólo entre estudiantes, manteniendo la distancia. Esto abre nuevas perspectivas a la educación a distancia. Se ha encontrado que, además, las nuevas posibilidades de interactividad pueden asimismo producir una nueva etapa en el futuro de este tipo de enseñanza, un modelo capaz de integrar la industrialización y una menos masiva y más personalizada relación, construyendo una 'comunidad académica virtual".

\section{Interactividad - Comunicación a través de Internet - Modelo fordista - Modelo post- fordista}

\begin{abstract}
Distance education is socially built as a kind of education without or with sorne particular interactivity. Because of this, interactivity is one of the central terms in the definition of distance education that carnes many meanings. And now, with the increasing use of Internet, interactivity can get other forms. Specially the web facilities the horizontal communication and the use of the image in the mediated meetings between learners and teachers or instructors, or only between students, rnaintaining the distance. This opens a new perspective to the distance education. It is found that new possibilites of interactivity can even so produce a new stage in the future of distance education, a model that could integrate the industrialization and a less rnassive and more personalized relation, building a "virtual academic community".
\end{abstract}

\section{Interactivity -. Internet communication - Fordist Model - Post-Fordist Model}

"Una hora de clase TV, una hora de baloncesto, de béisbol o de carreras, otra hora de transcripción o de reproducción de imágenes, y más deportes. Pero ha de saber que nunca hacemos preguntas o, por lo menos, la mayoría no las hace; no hacen más que 
lanzarte las respuestas, ¡zas!, y nosotros sentados allí durante otras cuatro horas de clase cinematográfica. Esto no tiene nada que ver con la sociabilidad"

Ray Bradbury, Fahrenheit 451

\section{UN SISTEMA EDUCATIVO EN TRANSFORMACIÓN}

La Galaxia Internet está transformando radicalmente la relación de la sociedad con la educación a distancia. De ser un tipo de enseñanza que rozaba lo periférico entre las percepciones sociales del sistema educativo, la ha alzado a horizonte futuro de todo tipo de enseñanza. La evocación del título de McLuhan (1972) no es gratuita, pues cabe prever que el creciente dominio de la comunicación digital va a crear otras formas de cultura, de relacionarse, de ser socialmente en el ámbito educativo. Un proceso que parece imparable hacia otras relaciones educativas. La propia comunicación electrónica ya se ha convertido en el paisaje cotidiano de la educación a distancia.

El término virtualización condensa actualmente el paso del dominio de un tipo de comunicación - escrita y/o telefónica- a otro tipo dominada por el uso de Internet. Se virtualiza un tema, una asignatura, un curso, un programa o toda una carrera universitaria; pero su significado frecuentemente va poco más allá de convertir lo publicado en papel - apuntes, temarios, guías, manuales, libros, etc.- a una publicación digital tras una página web de presentación. Quedándose aquí, podría decirse que Internet habría entrado en la educación a distancia; pero ésta aún no habría entrado metodológicamente en el medio y, sobre todo, en las lógicas comunicativas que se abren. Se exige así una mínima reflexión sobre las posibilidades de adaptación a estas nuevas lógicas de comunicación entre docente y alumno.

Reducir las posibilidades de cambio en la relación social y educativa a una sustitución de materiales puede conducir a no entender las transformaciones sociales que implica el dominio Internet. Caben dos formas de establecer la relación entre educación y las posibilidades de comunicación a través de canales electrónicos:

a) Adaptación de los medios electrónicos de comunicación al modelo educativo existente, que, salvo algunos aspectos, que pueden tener su importancia, ha de reconocerse como amplificación industrial (Peters, 1983) y masiva (Moya, 1976) del modelo tradicional, presencial. Internet se configura como un instrumento más, de carácter periférico o complementario, en un modelo de relación profesor-alumno que tiene por fuente básica la coincidencia espacial y temporal de ambos y una estática distribución jerarquizada de papeles donde el profesor explica o expone y el alumno recoge. El uso de los medios electrónicos de comunicación vendría a suplir la ocasional (enseñanza presencial) o sistemática (enseñanza a distancia) carencia de encuentro presencial. Su uso, por otro lado, estaría prácticamente concentrado en la exposición de materiales en la página web del profesor o asignatura y en las listas de correo electrónico, teniendo ambas prácticas un carácter imperativo.

b) Reformulación del modelo de relación entre profesor y alumno en función de las capacidades comunicativas que se derivan del uso de estos medios electrónicos. La pregunta ya no es para qué me sirve la tecnología, sino qué podemos hacer con las 
capacidades comunicativas de los nuevos dispositivos técnicos. No se trata de preguntarse tanto por el qué se puede comunicar ahora a través de estos medios, sino en qué medida se puede cambiar la relación comunicacional entre docente y alumnos. Esta segunda pregunta supone el paso de la preocupación por la virtualización — volcar los materiales en la red y, en todo caso, abrir otras vías de comunicación con el alumno, como el correo electrónico- al esfuerzo por la generación de relaciones interactivas en el proceso educativo.

La segunda posibilidad exige, al menos, una mínima reflexión y seguramente un importante cambio cultural del conjunto del sistema educativo. Pero se trata de un cambio que, de acontecer, viene impulsado por un contexto social que actualmente experimenta una importante convulsión, donde impera la sensación de que las cosas no van a seguir siendo como eran. Ni en la economía, ni en la política. ¿Por qué se va a resistir el sistema educativo a la posible transformación?

\section{CAPACIDADES COMUNICACIONALES}

Las capacidades comunicacionales a través de la web, en el ámbito de la enseñanza, son varias. Partiendo de la tripartita propuesta Tom Creed (2000), se puede distinguir entre:

a) Discurso privado: comunicaciones personales entre profesor y alumno sobre los contenidos de la materia u otros aspectos relacionados con la enseñanza de la misma. Tal discurso privado tiene su principal ubicación en el correo electrónico individualizado. Desde las instituciones de educación superior a distancia consolidadas, viene a sustituir y ser una síntesis de las comunicaciones telefónica y postal ordinaria, ya tradicionales. Se estructura principalmente desde la lógica consulta-respuesta. Desde tal capacidad comunicativa, pocos cambios se incorporan a la lógica comunicativa que se ha mantenido en los últimos años en la educación a distancia. Ahora bien, no es desdeñable el incremento de eficacia en el establecimiento de las comunicaciones, especialmente de las consultas del alumno al profesor.

b) Foro público: comunicaciones con un horizonte colectivo en cuanto a su construcción. Aun cuando los pasos iniciales en estos foros públicos pudieran venir del profesor, a modo de una pequeña exposición de cuestiones a tratar en el foro, su función es más estimular el debate y la participación de los alumnos que la transmisión de respuestas. La motivación se convierte en un elemento fundamental en la labor del profesor, pues ya no se trata sólo de motivar al alumno, sino de motivar la discusión. Se estructura desde una lógica dialógica, pudiéndose facilitar tal apertura a la participación con la posibilidad de garantizar el anonimato a quien así lo requiera -a través de utilización de claves especiales en el username- distinguiéndose así de las prácticas habituales de la enseñanza, tanto presencial como a distancia. El anonimato en el aprendizaje a través de la red se ha revelado como una notable fuente de igualdad (Collins y Berge, 1995; McComb 1994; Chester y Gwynne, 2000), aumentando las

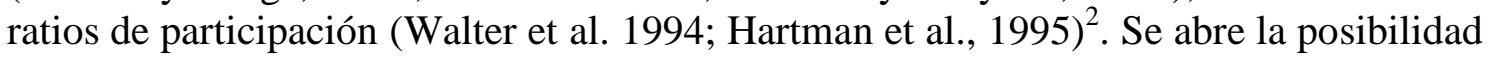
de generar un grupo participativo, y, así, de conseguir una comunidad académica, reutilizando el ya afamado concepto de comunidad virtual de Rheingold (1994). La poco atractiva sombra de una enseñanza individualizada, casi exclusivamente basada en el esfuerzo solitario, tiene aquí una medida que, al menos, la combate. 
c) Virtualización o depósito de materiales: lugar donde se encuentran los materiales (libros, apuntes), ya sea directamente en la web del profesor- asignatura, o facilitando los vínculos con otros lugares de la red relacionados con el contenido del curso. Se estructura desde la conocida lógica de la enseñanza a distancia en la que los soportes (materiales y canales) sustituyen la ausencia del profesor, poniéndose especialmente de relieve que sigue siendo la institución la que enseña a través de la gestión de los materiales, pues, como destaca García Aretio: "es la institución, más que el profesor, la que diseña, produce, distribuye, desarrolla o tutela el proceso de aprendizaje de los estudiantes" (García Aretio, 1 999b:48).

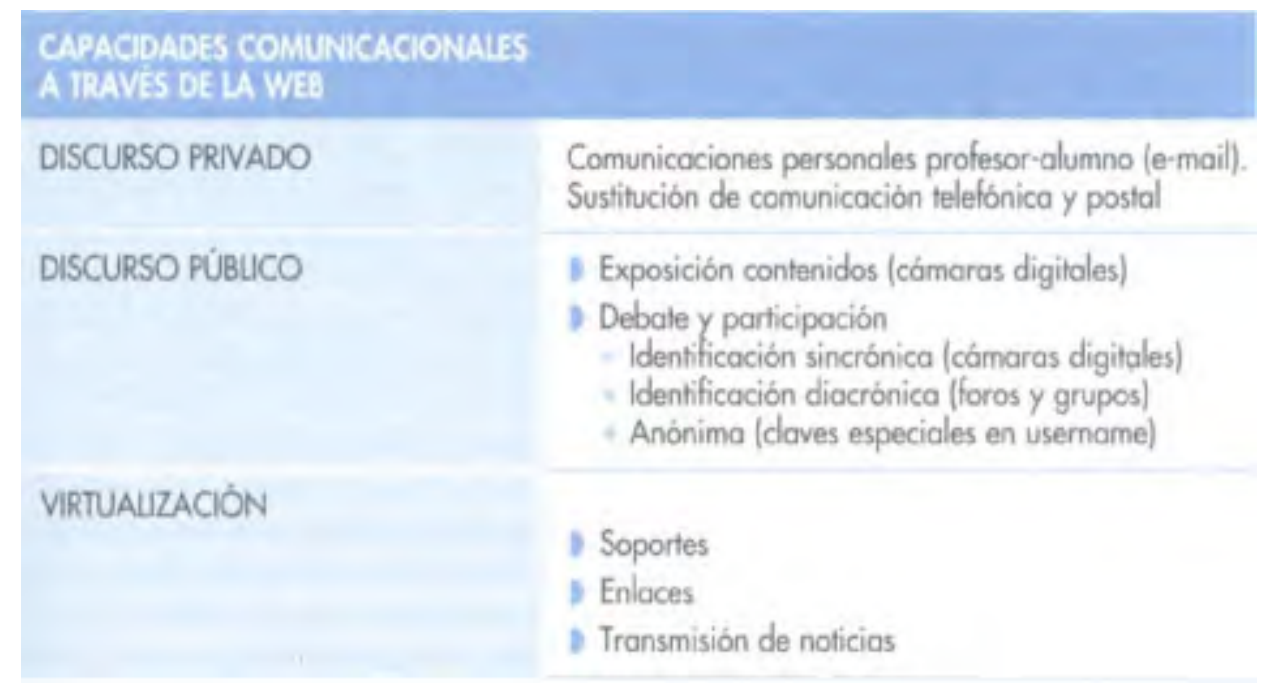

A las anteriores capacidades comunicacionales debe añadirse una cuarta que, aunque tenga un carácter instrumental-subsidiario, conviene recoger, ya que la experiencia nos señala su amplio uso, como es la de difusión de noticias. Tal capacidad se desarrolla principalmente a través de la lista de correo electrónico compuesta por los profesores y alumnos del curso o asignatura. Como b) y c), tiene un carácter colectivo, pues los receptores son varios homogéneamente; pero, a diferencia de b), no tiene el debate y la participación por objetivo; y, a diferencia de c), la recepción se privatiza, se introduce lo institucional en lo privado. De aquí que se haya señalado anteriormente con un rasgo imperativo. Esta cuarta capacidad comunicativa suele utilizarse para la transmisión de noticias, especificaciones, avisos, etc..

Puede considerarse que todas estas capacidades comunicacionales se encuentran en la actualidad satisfechas, en mayor o menor grado, con mayor o menor intensidad y velocidad, por diferentes medios, distintos a los medios electrónico-digitales. Pero lo importante es la posibilidad de integración de todas ellas en un mismo medio, no siendo baladí la cuestión del tiempo en la gestión de la enseñanza. Por otro lado, su potencial para algunas dimensiones toma, hoy, las características de lo infinito, con especiales repercusiones en el ámbito de la educación a distancia, generando las posibilidades de superar viejas barreras que aparecían como fuentes de deslegitimación de este tipo de enseñanza, frente a la presencial:

- Integración de lo individual y lo masivo (anónimo), superando la radical escisión entre comunicaciones individuales (consultas) y comunicaciones masivas (materiales, exámenes, notificaciones). Se abren espacios para la comunicación colectiva: una especie de «vuelta a clase»; pero una clase 
redefinida, capaz de superar algunas de las limitaciones (físicas y simbólicas) de la clase presencial.

- Integración de la interactividad y la interacción. Tradicionalmente, la enseñanza a distancia se ha configurado desde la ausencia de interactividad o se ha venido definiendo aquélla en función de la particular interactividad que genera (Holmberg, 1985), reconociéndose en la misma uno de los puntos básicos para una teoría integradora de la eduación a distancia (García Aretio, 1 999b:46). La interactividad constituía su carencia, era su valor ausente. La co-presencialidad física de la clase se convertía en garante de la interactividad, aun cuando, experiencia dixit, ésta se encontrase bastante limitada. Antes de seguir, hay que destacar que algunos consideran a la interactividad un mito de nuestro tiempo. No faltan críticos (véase Markus, 1987, 1990) e investigaciones específicas que muestran su escasa relación con el aprovechamiento académico (Kelsey, 2000), subrayándose que se trata de una demanda de unos pocos alumnos muy activos. Además, debe reconocerse que se ha amplificado en exceso y comercialmente este concepto. Pero, a pesar de ello, la interactividad abre un panorama distinto en la enseñanza a distancia, que es en el que nos vamos a demorar en los apartados siguientes.

- La integración de las distintas capacidades comunicacionales señaladas transforma el significado de aprender, que no puede seguir significando transmisión-recepción de información. El problema no es la exposición o transmisión de información -desde los libros a los apuntes se encuentran en la red- sino la creación de información. Como dice Bili Gates, toda información existente se encuentra: «a un par de clics de ratón» (Gates 2000:45). Hay que enseñar a crear información, no dedicando la relación profesor-alumno a establecer una cadena en la mera transmisión. Dentro de la integración de las capacidades comunicacionales, hay que destacar el nuevo papel que adquiere la imagen en el intercambio comunicativo. Hasta ahora, el grueso de las comunicaciones entre profesor y alumno, en la educación a distancia, se ha apoyado en la escritura. Puede decirse que, salvo en el ocasional uso de videoconferencias con una estructura un tanto rígida, la falta de imagen en la relación entre profesor/evaluador y alumno/evaluado era una de las características diferenciales de este tipo de educación. A través de Internet y el abaratamiento, mejora técnica y extensión de las cámaras digitales, el profesor y los compañeros pueden estar, con su imagen, en la pantalla del ordenador o la televisión (digital) domésticos, y a la inversa. A su vez, los alumnos pueden estar ante el profesor cuando intervengan.

Interactividad se ha convertido en un término tan usado que ha pasado de señalarse como valor añadido a generar sospechas allí donde se aplica. En primer lugar, conviene separar entre interactividad e interacción. Ésta es la acción que se ejerce recíprocamente entre dos o más objetos, agentes, fuerzas, funciones, etc. (Real Academia Española). Desde nuestro punto de vista, la interacción es algo pasado, lo que permite el registro. Tal rasgo permite concebirla sin excesiva dificultad en un elemento susceptible de evaluación, pues todo lo registrado permite ser evaluado. Algo es interactivo en la medida que ejerce la interacción (Seco, Andrés y Ramos, 1999). Sin embargo, la interactividad nos habla de la facultad de obrar conjuntamente, de que la interacción se produzca. Nos habla de las capacidades de interacción, lo que implica aspectos sociales. 
La interactividad en la clase presencial no es tan susceptible de evaluación directa. Ahora bien, y aquí radica uno de los basamentos de esta propuesta, en la medida que se registre -y evalúe- la interacción y las habilidades didácticas para producir interacciones generan interactividad, transformándose progresivamente así la relación entre profesor y alumno. Es decir, la concreción de la posibilidad técnica amplía el espacio de una cultura de la interactividad.

Antes de introducirnos en algunas consecuencias de la asunción de la interactividad, han de distinguirse niveles o tipo de la misma. Hay una interactividad simple, que es la cercana a la lógica preguntas-respuestas más frecuentes (FAQ). Es una interactividad mecánica, que, de hecho, ya recogen los programas de ordenador. Permanecer aquí sería aceptar al profesor como una máquina de enseñar (Skinner, 1968). La interactividad compleja que se propone está fundada en los sistemas autopoiéticos, generadora de más interactividad. La huella de la interacción (registro) genera interactividad, especialmente en un subsistema, como el educativo, donde el registro constituye la base, como muestra el amplio uso del curriculum y los certificados.

Ha de reconocerse que cada tipo de comunicación, en la medida que se desenvuelve en una articulación de espacio, tiempo y relaciones sociales, tiene su interactividad. Siguiendo con la clasificación de Creed para la web: el discurso privado, en cuanto se sigue apoyando en la lógica consulta-respuesta, apenas genera interactividad; la virtualización genera, a lo sumo, una interactividad diacrónica, aun cuando aparece más condenada a una estructura de mera interacción institucional: exposición de materiales y noticias en la red y respuesta en los exámenes. Es la que Moore (1989) denomina como interacción con los contenidos de la materia de estudio, siendo la más antigua, desde la perspectiva de la historia de la educación a distancia, y la que, en definitiva, puede considerarse como imprescindible, pues sitúa al aprendiz ante su materia. El uso de los foros públicos aparece con el mayor potencial de interactividad. Abre, además, la posibilidad de la interacción entre alumnos, es decir, la interactividad horizontal, ya sea con o sin presencia en tiempo real de un profesor o tutor, como subraya el propio Moore (1989:4), facilitando la creación de grupos, con su propia dinámica.

\section{LA INTERACTIVIDAD: UN CONCEPTO COMPLEJO}

La diferencia entre interactividad e interacción y los diferentes grados de concreción de aquella, nos pone sobre la pista de las dificultades de su aprehensión. Como señala Moore (1989:1), la interacción es otro de los términos con muchos significados y subsignificados. Si a esto se añade la ubicación en un contexto informático, el significado del concepto se complica.

La principal dificultad se halla en la proyección del concepto articulando distintos niveles lógicos, como son el de potencialidad - algo es interactivo si ofrece posibilidades de interacción- y el de concreción, pues algo es interactivo si realmente pone en juego la interacción, hace, al menos, jugar a la interactividad. Ha de articular la competencia y la actuación. Lo cual parece irrenunciable, si no se desea caer en la interacción.

La interactividad ha sido abordada sociogenética y ontogenéticamente por Habermas (1989: 166 y ss.), pues la competencia interactiva se forma en el yo simultáneamente con las competencias cognitivas y lingüísticas. Es decir, ha de acudirse a un marco 
teórico relevante para observar el lugar de la interactividad, lo que ya pone en evidencia sus profundas implicaciones.

Destaca de la propuesta de Habermas la extensión que hace de la pragmática lingüística y la fuerza ilocucionaria de los actos de habla, pues interesa rescatar la idea de cómo el habla deriva en vínculo, es decir, cómo la actuación se convierte, a su vez, en competencia. Pero el habla requiere, a su vez, una estructura comunicativa: "el hablante asume la garantía de que en, consecuencia con su emisión, cumplirá determinadas condiciones: por ejemplo, dar por zanjada una pregunta cuando se dé una respuesta satisfactoria; retirar una afirmación cuando quede claro que es falsa; insistir en una exigencia cuando no sea observada, etc.. La fuerza ilocucionaria del acto de habla consiste, por tanto, en que puede mover a un oyente a poner a la base de su acción la suposición de que la oferta del hablante es seria" (Habermas, 1989:173). El acto de habla se mueve en suposiciones sobre la relación social. Es decir, sobre la estructura comunicativa presente y las posibilidades de relación. Habrá interactividad en un marco que genera interactividad. La mera posibilidad técnica no basta. Desde el punto de vista técnico material, hay capacidad para la interactividad en la relación entre un juez y un juzgado en una sala de tribunales; pero las relaciones sociales apenas permiten su desarrollo. Como subrayan Vrasidas y Mclsaac (1999) en sus conclusiones para el campo de la educación a a distancia, las relaciones de poder son un mediador de enorme peso para el ejercicio de la interacción, pues, cuando están muy presentes se bloquea la interactividad.

El problema de seguir el discurso teórico de Habermas es que asimila como comunicación distorsionada toda aquella dirigida por acciones estratégicas. De hecho, acción comunicativa y acción estratégica aparecen como conceptos opuestos. Sin embargo, ha de reconocerse que la relación educativa está dominada por acciones estratégicas. No se trata de alcanzar consensos sino de enseñar y certificar, por un lado, y aprender y obtener el título, por el otro. Hay más intercambios estratégicos, que la propia noción de programa señala, que una especie de fusión discursiva. Pero ello no ha de suponer condenar la interactividad a una especie de simulacro o de examen continuo bajo la atenta mirada del profesor. También puede caber la crítica y el examen del profesor por parte de los alumnos. No es imposible sino simplemente algo distinto desde la actual cultura dominante en los procesos educativos. Hay una estructural relación de dependencia —el alumno depende del aprobado del profesor- que impide, en un principio, la acción comunicativa tal como la entiende Habermas; pero no impide la interactividad y que ésta, además, sea estratégicamente eficaz a los intereses de ambos agentes, de quienes quieren enseñar y de quienes quieren aprender.

La interactividad refuerza la estructura comunicativa y la relación social. Podría decirse que las hace "más calientes". Pero se limita a reproducir ampliada- mente la estructura comunicativa, no a crearla. Amplía e intensifica los vínculos, eso sí, regenerando el marco y la competencia interactiva.

La estructura que pone en juego el uso de Internet es interactiva; pero para que sea una estructura intensamente comunicacional, donde operen los niveles semántico e intersubjetivo, hace falta algo más que un medio. Hace falta un contexto comunicativo apropiado, un consenso previo sobre los derechos y deberes de los agentes y, en especial, sobre los contenidos y aspectos de los intercambios. El uso de la web facilita la comunicación, incluso a los más tímidos (Ambrojo, 2000:21), especialmente por la 
posibilidad de anonimato. Pero no crea el marco de comunicación, lo que supone un consenso previo o, más explícitamente, una cultura de la comunicación interactiva en el proceso de comunicación.

La interactividad tiene, por lo tanto, dos niveles:

- La competencia técnica-comunicacional, la facilitada por las características de los medios, que, en este caso, Internet facilita.

- La competencia social que permite o impide la concreción de la interactividad. De hecho, ésta lalla si no hay cultura de la interactividad (Gonzales 1989, Rafaeli 1988). De hecho, como se verá más adelante, las resistencias no se encuentran sólo en quienes han de realizar el mayor esfuerzo de adaptación para poner en funcionamiento la interactividad, los docentes, sino en unos alumnos que, por ejemplo, suelen interesarse poco o nada por los chats puestos a su disposición en las asignaturas, ya que su percepción estratégica no los incluye como medios para el aprobado o el logro del título.

Hace falta la articulación de los dos niveles para que se dé la interactividad.

\section{TRANSFORMACIÓN EN LA RELACIÓN}

La concreción de la interactividad implica un grado de sociabilidad, siguiendo las palabras del novelista de ciencia ficción Ray Bradbury expuestas en el encabezamiento de este escrito. La estructuración de otro tipo de sociabilidad es lo que convierte la interactividad en concepto clave para el futuro de la educación a distancia, haciéndola radicalmente diferente $y$, a la vez, frontalmente competitiva con la enseñanza presencial.

Una relación apoyada en la interactividad transforma la habitual vinculación entre profesor y alumno. Se abordan sólo algunos aspectos:

- Aprender no estará basado en la memoria, algo que se ha dicho muchas veces, sino en la crítica: en la capacidad de aumentar el flujo comunicacional, produciendo más interacciones, a partir de la introducción de atractoresgeneradores en el mismo, que repercutan en los demás participantes del foro, en las comunicaciones públicas, o de los alumnos, en los discursos privados. Claro está, tal fundamentación en la crítica puede ir más allá de los contenidos, proyectándose sobre la propia figura del profesor, de manera que la distante jerarquía de éste se verá menguada. La diferencia entre profesor-alumno no puede estar basada sólo en aspectos institucionales, estáticos, sino que también es procesual, ha de renovarse en el proceso - por lo que ya es una fuente para la mejora- desde su actuación comunicativa. Esto exigirá un importante esfuerzo de adaptación en algún sector del profesorado que actualmente se encuentra en la educación a distancia. Pasar de los materiales y las técnicas para hacer materiales a las técnicas de contacto y comunicación interactiva no se hace de la noche al día y van más allá del uso de programas de ordenador. Esto es sólo la carcasa. Es más, el profesor y sus técnicas para la generación de dinámicas grupales adquieren renovada relevancia.

- Paso de una relación en la disciplina a una relación en el proyecto y la resolución de problemas. El examen seguramente tendrá menos relevancia, 
dejando lugar a los ejercicios, las simulaciones y los casos (por ejemplo, cada semana el profesor presenta un caso, que ha de resolverse por los alumnos en la red, pudiendo estos preguntar a través de un chat, foro o individualmente) ${ }^{2}$.

- Deja de tener sentido la acreditación en base al tiempo, a las horas, para hacerlo la propia actividad (el tiempo queda en relativo suspenso).

- El horizonte es formar una comunidad de conocimiento (Kerckhove, 1999), haciéndose más integradamente colectivo el conocimiento, produciéndose una nueva concepción de lo que es «una clase». Es más, si las nuevas tecnologías de la información y la comunicación no sirven para fundamentar una comunidad académica, la mera virtualización de la educación a distancia estaría condenada al fracaso, como subraya Daniel (1999:33).

La integración de la interactividad supone una nueva etapa de la educación a distancia, que va más allá de la incorporación de las tecnologías de la comunicación y la información. Si cabe hablar de tres generaciones de la educación a distancia: correspondencia, telecomunicación y telemática (Garrison 1985, 1989; García Aretio 1 999) esta nueva etapa podría considerarse una profundización de la tercera o realmente una nueva cuarta etapa, pues, siguiendo a Martín y Ahijado sobre la evolución de la enseñanza a distancia: "El desarrollo de las instituciones ha estado vinculado, en cierta medida, con su capacidad y compromiso para incorporar las aportaciones tecnológicas como elementos de avance y renovación rnetodológica. De manera progresiva se ha transitado, desde una perspectiva de integración y complementación de soluciones tecnológicas, por distintas generaciones relacionadas la primera con el uso casi exclusivo de los materiales impresos, la segunda con la utilización además de los lenguajes y medios audiovisuales hasta llegar a lo que ya empieza a conocerse como la tercera generación de las tecnologías de la educación a distancia, las nuevas tecnologías de la información y comunicación" (Martín y Ahijado 1999:12). A favor de su consideración como cuarta etapa, diferenciada de una tercera, se encuentra el hecho de que cabe interpretar las tres primeras como concreciones diferenciadoras de una misma lógica desde el punto de vista de la sociabilidad que ponen en marcha. Se trata de una sociabilidad masiva, donde lo importante ha sido llegar al mayor número de personas de manera homogénea, lo que supone una serie de elementos estandarizadamente rígidos que son un obstáculo para la propia interactividad.

Las transformaciones señaladas tienen repercusiones en el modelo de educación superior a distancia. La objetivización de la interacción requiere cierta formalizaciónestandarización y, en definitiva, es proclive a integrarse en el modelo industrial que rige las grandes universidades de enseñanza a distancia. De esta manera, la relación profesor-alumno también puede «industrializarse».

La enseñanza a distancia es una enseñanza industrial. Sin este prerrequisito tal vez no tenga sentido. Al menos, sentido económico, que es lo que está moviendo a otras instituciones tradicionalmente presenciales a acercarse a la enseñanza a distancia. Sin embargo, con la incrustación del binomio interactividad compleja/evaluación3, no puede hablarse del mismo tipo de industrialización. De la industrialización de masas se puede estar dando el paso hacia la industrialización flexible y, en buena medida, hacia una industrialización con notables estándares de calidad para mantener la supervivencia. 
En el sistema educativo a distancia industrial de masas, todo está basado en el examen. Todo se dirige al examen. Desde las unidades didácticas, al tutor, pasando por el material y los medios. Desde las guías didácticas a las intervenciones en radio.

Son varios los puntos en que es posible la comparación de diferencias entre un modelo industrial y otro. Por señalar sólo uno: en la versión fordista de la planificación, se planifica, siendo esta la obsesión de Peters (1998); sin embargo, la didáctica de la interactividad es abierta, infinita. Es más, no es una didácticá de las respuestas, sino de preguntas que generan preguntas. Otros cambios pueden observarse en el siguiente cuadro:

\begin{tabular}{|c|c|c|}
\hline $\begin{array}{l}\text { MODELOS DE } \\
\text { ENSENANZA A } \\
\text { DISTANCIA }\end{array}$ & $\begin{array}{l}\text { INDUSTRIAL } \\
\text { DE MASAS } \\
\text { (FORDISTA) }\end{array}$ & $\begin{array}{l}\text { NDUSTRIAL } \\
\text { FISXIBLE-FRACTAL } \\
\text { (POSTRORDISTAL }\end{array}$ \\
\hline $\begin{array}{l}\text { Principales } \\
\text { elementos }\end{array}$ & $\begin{array}{l}\text { Materiales referenciales y } \\
\text { cerrados (las guías de } \\
\text { orientación han de tender al } \\
\text { cierre de lo no cerrado) y } \\
\text { exámenes }\end{array}$ & $\begin{array}{l}\text { Materiales abiertos y criticos, en } \\
\text { constante renovación, donde } \\
\text { pueden incorporarse las criticas } \\
\text { de los alumnos. }\end{array}$ \\
\hline Lógica dominante & $\begin{array}{l}\text { Planificación-previsión. El } \\
\text { calendario ocupa un lugar } \\
\text { central La institución genera la } \\
\text { dinámica. }\end{array}$ & $\begin{array}{l}\text { Decisión en situaciones y grupos, } \\
\text { con relativa autonomia. El grupo } \\
\text { adapta la dinámica } \\
\text { preestablecida por la institución }\end{array}$ \\
\hline Contexto social & $\begin{array}{l}\text { Rutinas, De cara al aprendizaje, } \\
\text { se acentúa la importancia de la } \\
\text { adquísición de hábitos de estudio } \\
\text { por parte del alumno. }\end{array}$ & $\begin{array}{l}\text { Ruptura continua. Especialmente } \\
\text { interesa aprender a aprender, en } \\
\text { la configuración de una } \\
\text { conciencia crítica creativa. Esta } \\
\text { afirmación, que parece un tanto } \\
\text { idealizada, se ho convertido en } \\
\text { un recurso económico crucial. }\end{array}$ \\
\hline $\begin{array}{l}\text { Papel de la } \\
\text { institución }\end{array}$ & $\begin{array}{l}\text { Garantía de materiales y de los } \\
\text { exámenes }\end{array}$ & $\begin{array}{l}\text { Garantio de los profesores. La } \\
\text { institución es la que garantiza un } \\
\text { estándar de calidad de los } \\
\text { profesores }\end{array}$ \\
\hline
\end{tabular}




\begin{tabular}{|c|c|c|}
\hline $\begin{array}{l}\text { Lugar de los } \\
\text { alumnos }\end{array}$ & $\begin{array}{l}\text { Anónimo-masivo. Demanda de } \\
\text { estudios superiores }\end{array}$ & $\begin{array}{l}\text { Participantes identificados. } \\
\text { Demanda de habilidades. Se } \\
\text { conforman como grupo, frente y } \\
\text { junto al profesor, y no como } \\
\text { átomos de masa. }\end{array}$ \\
\hline $\begin{array}{l}\text { Papel de los } \\
\text { profesores }\end{array}$ & $\begin{array}{l}\text { Secundario: un complemento. } \\
\text { Pueden ser meros } \\
\text { "administradores/gestores» de } \\
\text { los contenidos de los materiales } \\
\text { Examinodores. } \\
\text { Garantizar la transimisión del } \\
\text { conocimiento (ofrecer materiales } \\
\text { y evaluar) }\end{array}$ & $\begin{array}{l}\text { Principal: la relación con la } \\
\text { institución pasa por el profesor. } \\
\text { Asesores-evaluadores. } \\
\text { Facilitodores del aprendizaje. } \\
\text { Asesorar en la loma de } \\
\text { decisiones (disciplinares, } \\
\text { curriculares) (Bricall: 195) } \\
\text { El profesor recobra protogonismo. }\end{array}$ \\
\hline $\begin{array}{l}\text { Relación } \\
\text { docente/discentes }\end{array}$ & Jerárquico estático & Dinámica participativa \\
\hline Evaluaciōn & Reducida al examen & $\begin{array}{l}\text { Continua, a través de la } \\
\text { interacción }\end{array}$ \\
\hline $\begin{array}{l}\text { Lógica dominante } \\
\text { de comunicación }\end{array}$ & $\begin{array}{l}\text { Examen y encuesta: pregunta- } \\
\text { respuesta }\end{array}$ & $\begin{array}{l}\text { Dialógica: hablar alrededor de } \\
\text { algo, aunque haya sido ya dicho }\end{array}$ \\
\hline $\begin{array}{l}\text { formas de } \\
\text { comunicoción } \\
\text { dominantes }\end{array}$ & $\begin{array}{l}\text { Unidireccionales públicos (guias) } \\
\text { y privados (cartas) y } \\
\text { bidireccionales privados } \\
\text { (teléfono) }\end{array}$ & $\begin{array}{l}\text { Bidireccionales públicos (chats en } \\
\text { Internet) }\end{array}$ \\
\hline Tipos de centros & Megauniversidades & $\begin{array}{l}\text { Centros de tamaño medio (Las } \\
\text { actuales escuelas de negocio } \\
\text { aparecen como modelo) }\end{array}$ \\
\hline $\begin{array}{l}\text { Melavalidez a } \\
\text { volidez pragmática } \\
\text { fundamental, una } \\
\text { vez logrodo el título }\end{array}$ & $\begin{array}{l}\text { Para oposiciones } \\
\text { Gran Administración. } \\
\text { Mercado laboral estable y muy } \\
\text { regulado }\end{array}$ & $\begin{array}{l}\text { Profesional autónomo y abierto. } \\
\text { Mercodo laboral dinámico, } \\
\text { tendente a la reducción de la } \\
\text { regulación }\end{array}$ \\
\hline Función & $\begin{array}{l}\text { Formación de actitudes (en linea } \\
\text { con la Universidad tradicional) }\end{array}$ & $\begin{array}{l}\text { Formación de aptitudes } \\
\text { (aprender a hacer, en conexión } \\
\text { práctica) }\end{array}$ \\
\hline $\begin{array}{l}\text { Tipo de relación } \\
\text { molivada }\end{array}$ & $\begin{array}{l}\text { Individual jerárquica- } \\
\text { subordinada }\end{array}$ & $\begin{array}{l}\text { Grupal competitiva (entre grupo } \\
\text { y profesor y intragrupo) }\end{array}$ \\
\hline
\end{tabular}

Claro está, la síntesis expositiva, acentuada por la utilización del cuadro, refuerza las oposiciones, de manera que, en algún caso puede interpretarse como excluyente lo que es sólo cuestión de grado, como ocurre con la diferencia entre la función del modelo fordista, más volcado, como el conjunto del sistema educativo superior, a la creación de actitudes y, de manera subyacente, de una especie de cuerpo, "el de los universitarios", como recoge el lenguaje popular, al modelo post-fordista, que aparece más inclinado a la formación práctica.

El paso de una etapa a otra no ha de estar exclusivamente regido por el costo sino que el segundo se impondrá al primero por el rendimiento pedagógico. El grupo que se constituye a través de la relación con la asignatura, pudiéndose precisamente hablar de grupo, se convierte en fuente de aprendizaje - se aprende de los demás- y en grupo de control del propio proceso de aprendizaje, siendo él mismo el que observa los resultados 
(La Rose, Gregg y Eastin, 1998). Puede decirse que el modelo post-fordista es más reflexivo en su evaluación, pues, además de los costes por unidad —base del modelo industrial- incorpora el grado de satisfacción de los agentes de la comunidad académica.

La utilización de la imagen puede ayudar enormemente a la generación de tal comunidad académica. A través de la cámara, la difusa comunidad "virtual" parece adquirir mayor realismo. Es ésta la virtud de lo virtual, la constitución de lo lejano. Se constituye una clase y una relación didáctica donde antes sólo había materiales. La imagen facilita otra sociabilidad.

Al respecto, hay que señalar la existencia de otra forma de interactividad que también utiliza la imagen, como la que deriva de la utilización de vídeos interactivos (Bennington, 2000), algo que cierra relativamente la interactividad aquí propuesta. Una especie de camino intermedio entre la simple virtualización (transformación digital estática de los materiales) y la interactividad.

Por otro lado, hay aspectos que se mantienen. Así, la garantía de objetividad en la evaluación, con que aparecen los exámenes en la educación a distancia, donde sólo se valora un registro (lo escrito por el alumno en el examen), se transforma en la posibilidad de registrar las mismas interacciones en la memora del ordenador, sean meramente escritas (e-mail, chats, foros) u orales (registro audio).

\section{UN CAMBIO CULTURAL}

La evocación a la interactividad lleva demasiado tiempo en el campo educativo como para no preguntarse por las razones de su ausencia en el mismo. El problema se encuentra precisamente en su potencial. No sólo implica un cambio técnico sino también un cambio cultural. Como ya se sabe, los cambios culturales son bastante más lentos que los meros cambios técnicos. En muchos campos, como en periodismo, no se explota el potencial de interactividad de los medios (véase Schultz, 1999). El campo de la enseñanza, por abierto y activo que sea, no supone una excepción.

El informe Dearing sobre la enseñanza superior en Gran Bretaña otorga a la interactividad la categoría de meta del sistema educativo. Pero su presencia ya se encontraba en las primeras reflexiones sobre las consecuencias de la extensión de los medios digitales en la sociedad, como las expuestas en el informe de Nora y Mmc: «Poco a poco se desarrollará una relación diferente, hecha de diálogos y reiteraciones sucesivas, que cada vez desbrozarán un camino original. Esta mutación, llevada a sus consecuencias extremas, producirá una metamorfosis de la pedagogía ¿Qué significarán entonces los conceptos de programas escolares, los cursos preestablecidos y las separaciones entre las materias, si el ritmo y la naturaleza de la enseñanza variarán de un alumno a otro?" (Nora y Mmc, 1982:93).

Las primeras resistencias se encuentran en el propio campo educativo. El mismo informe Deanng nos habla de dos tipos de resistencias entre los profesores. Por un lado, las resistencias de algunos sectores hacia el uso de las tecnologías de la información y la comunicación (punto 8.27 del informe nacional). Por otro lado, resistencias a la misma interactividad, al cambio de relación que implica y el hecho de que la mayor parte del profesorado universitario no sabe gestionar la interactividad (punto 8.17 del informe nacional). Por último, la interactividad supone pedir a los docentes un esfuerzo especial 
de adaptación, de puesta al día, que, además, desde la perspectiva meramente laboral no tiende a aparecer con rasgos atractivos. Es más, surge el fantasma de un profesor multimedia que, a la manera del periodista multimedia que observa cómo se le solicita mayor esfuerzo - más horas- para que el producto de su trabajo aparezca en todos los medios de la gran corporación mediática (diarios, revistas, radios, televisiones), se ve en la necesidad de alargar sus jornadas para contestar unas demandas de los alumnos que ahora le vienen por muy diversas fuentes, y de muy diferentes maneras. Es decir, el desarrollo de la interactividad en la educación a distancia exige un cambio de la relación de números de alumnos por profesor. En términos de costo, seguirá siendo ventajosa para la educación a distancia, en su comparación con la educación presencial; pero aparecen como incompatibles las abultadas ratios de números de alumnos por profesor, que el modelo industrial y masivo de la educación a distancia permite, para desarrollar la interactividad.

Siendo claves las resistencias de los profesores, ha de tenerse también en cuenta la matriz cultural de la que parten, tanto profesores como alumnos. La interactividad no sólo genera creatividad, sino que deriva de un marco cultural que promueve la actividad y creatividad de los sujetos. Hofstade describe cómo en las culturas autoritariastradicionales y con el proceso educativo centrado en el profesor, las intervenciones del alumno son pautadas o, en general, restringidas. Enculturas con una distancia jerárquica menor: «Los alumnos intervienen en clase sin necesidad de que se les invite a ello y deben hacer preguntas cuando no comprenden aleo» (Hofstede, 1999:77). Para el caso de los alumnos universitarios, ha de tenerse en cuenta que han crecido en una escuela que no suministra las habilidades para la participación en el proceso de enseñanza.

También ha de tenerse en cuenta que, en los países económicamente desarrollados, la extensión del uso de la telemática ya no se debe tanto a razones financieras, como a razones de índole cultural.

La necesidad de cambio cultural puede ser un obstáculo; pero, cuando se experimentan momentos históricos tendentes al cambio, también puede ser un estímulo para que unos subsistemas de la sociedad más dinámicos empujen a otros. Actualmente, es la sociedad la que demanda el cambio a la educación superior, como pone de manifiesto la proliferación de informes nacionales sobre la misma.

La extensión de la evaluación en el sistema educativo superior en los países desarrollados, en general, y en España, en particular, también es indicativo de la experimentación de ciertos cambios culturales en el interior de tal sistema. Pero la extensión de las tecnologías de la comunicación y la información y la cultura de la evaluación son dos procesos que están yendo paralelos; pero no de la mano. Se resume en la contabilización del número de ordenadores o incluso acceso a Internet por cada grupo y categoría de trabajadores de la institución, como pone de manifiesto la Guía de Evaluación del Plan Nacional de Evaluación (Consejo de Universidades).

Pero tal vez lo peor, es lo reducido del campo en el que se ve la posible influencia de estos medios de cara al futuro:

- Técnicas para gestionar el proceso de aprendizaje y asesoramiento.

- Evitación de la duplicación de tareas administrativas.

- Admisión de estudiantes. 
Los dos procesos, evaluación y desarrollo de la interactividad, parecen condenados a entenderse, yendo más allá de las prácticas de gestión institucional. No obstante, desde las mismas instituciones universitarias, la articulación de ambos procesos puede caminar en su beneficio.

La interacción mediada por medios de comunicación digitales puede registrarse. Todo el flujo comunicacional entre profesor y alumnos o entre alumnos es susceptible de acumulación, contabilización, evaluación y análisis, principalmente de las sintaxis que se producen. Puede obtenerse así un rico material para evaluar -reflexiva e institucionalmente- la actividad del profesor, como para que la institución y el profesor puedan evaluar la situación de cada uno de los alumnos en un momento específico. De alguna manera, la caja negra del aula se abre. Es cierto que esto tal vez añada resistencias en el profesorado, a las ya existentes con respecto al uso de los medios electrónicos, la interactividad y la evaluación; pero la tendencia hacia una evaluación constante y transparente, hacia la cultura de la evaluación parece muy fuerte.

Sobre este aspecto, ha de señalarse que la interactividad registrada en interacciones supera en objetividad a la interactividad que se produce en el interior del aula tradicional. En ésta, el registro de la interacción se encuentra exclusivamente mediado por la subjetividad del profesor a la hora de la evolución, aun cuando se trate de esa objetivización subjetivada producto de la socialización en un contexto y un campo (Bourdieu, 1988). Se trata de pasar de la objetivización subjetivada a la subjetivización de los componentes con poder de decisión en el sistema- objetivada en protocolos de evaluación, lo que, al menos, siempre es revisable desde un observador externo. Pero tal vez lo más importante es que la doble evaluación, del alumno por el profesor y del profesor por el alumno, se hace constante y dinámica. Asimismo, la recíproca retroalimentación se convierte en fuente para las respectivas reflexividades. Tal tipo de evaluación constante e interactiva está inscrita en las propuestas del denominado informe Bricail: «Esta evaluación es un proceso que debería ir más allá de la mera y tradicional rutina de calficación de estudiantes, para pasar a ser un sistema continuado de medida de la calidad del propio sistema pedagógico» (Informe Universidad, 2000: 189).

Por lo tanto, la incorporación de la interactividad compleja deriva de un cambio cultural; pero, a su vez, se convierte en germen de una cultura para el cambio. De aquí que la profundización en este sentido de la cultura de la evaluación en el sistema educativo se acentúe como evaluación hacia el cambio.

\section{CONCLUSIONES}

La denominada virtualización apenas supone un primer paso en la incorporación de los medios electrónicos en la educación a distancia. La plena integración de los medios digitales de información y comunicación a la enseñanza a distancia pueden transformar radicalmente la relación entre profesor y alumno y, con ello, el modelo en el que básicamente se ha sustentado este tipo de enseñanza. Para ello, juega un papel clave la articulación entre interactividad compleja y evaluación continua. De hecho, abogar por la interactividad es devolver el interés por el proceso comunicativo en la relación educativa. 
De esta manera, la extensión del uso de Internet va a suponer una revolución en la enseñanza a distancia que, en sí misma, ya ha supuesto una revolución en la propia concepción de la enseñanza. Por último, consignar que, radicado en la interactividad digitalizada, la didáctica de la enseñanza a distancia, el modelo de la enseñanza a distancia puede desprenderse ya, casi definitivamente, de los modelos de la enseñanza presencial, que son los que le han servido de modelo: el modelo expositivo, trasladado de la voz del profesor a la letra del libro.

La profundización en el concepto de interactividad conduce a observar que la concreción de su grado más intensos y, sobre todo, de carácter público, posibilitando la comunicación horizontal entre profesor-alumno y entre alumnos, facilitado técnicamente por los medios digitales ya presentes, conduce a otro modelo de educación a distancia. Un modelo que se ha denominado industrial post-fordista, en cuanto mantiene algunos aspectos del carácter industrial de la enseñanza a distancia; pero, a su vez, exige una mayor y más profunda adaptación a las diversas demandas y características del alumnado, lo que le impide ser masiva. De hecho, se trata de adaptar el propio modelo de la educación a distancia al modelo industrial ideológicamente dominante en las sociedades avanzadas.

\section{REFERENCIAS BIBLIOGRÁFICAS}

AMBROJO, J.C. (2000): "La 'web' transforma los métodos pedagógicos de Stanford", en El País (suplemento Ciberpaís), 24 de febrero de 2000.

BENNINGTON, T, L. (2000): "Mediated Perceptions: Contributions of Phenomenological Film Theory to Understanding the Interactive Video Experience", Journal of Computer Mediated Comunication, vol. 5 (4), junio.

BOURDIE, P. (1988): El sentido prdctico, Madrid: Taurus.

CHESTER, A., y G. GWYNNE (2000): “Online Teaching: Encouraging Collaboration through Anonymity", en Journal of Computer Media ted Comunication, vol. 4 (2), diciembre.

COLLINS, M. y Z. BERGE (1995): "Introduction: Computer-mediated communications and the online classroom in higher education", en Z. L. Berge y M. P. Collins (eds.), Computer mediated communication and the online classroom: Higher education (vol.2), Nueva Jersey: Hampton

Press.

CREED, T. (2000): «Extending the Classroom Walls Electronically» en http://www.users.csbsju.edu

DANIEL, J. (1999): "EVa a ser la macro-universidad el modelo de formación académica superior en el nuevo milenio?", en E. Martín Rodríguez y M. Ahijado Quintillán (Coord.), La educación a distancia en tiempos de cambios: nuevas generaciones, viejos

Ed.

de conflictos,

Madrid: la

Torre. 
GARCÍA ARIETO, L. (1999a): "Historia de la Educación a Distancia", en Revista Iberoamericana de Educación a Distancia, vol. 2(1), junio, págs. 11-41.

GARCÍA ARIETO, L. (1999b): "Fundamentos y Componentes de la Educación a Distancia", en Revista Iberoamericana de Educación a Distancia, vol. 2 (2), diciembre, págs. 43-62. GARRISON, D.R. (1985): "Three generatins of technological innovation in distance education”, en Distance Education, 6, pp. 235-24 1.

GARRISON, D.R. (1989): Understanding distance education, Londres: Routledge.

GATES, B. (2000): Los negocios en la era digital, Barcelona: Plaza y Janés.

GONZÁLES, H. (1989). "Interactivity and feedback in Third World development campaings", en Critical Studies in Mass Communication, 6 (3), pp. 295-314.

HABERMAS, J. (1989): Teoría de la acción comunicativa: complementos y estudios previos, Madrid: Cátedra.

HARTMAN, K., C. M. NEUWIRTH, S. KIESLER, C. COCHRAN, M. PALMQUIST y D. ZUBROW (1994): "Patterns of social interaction and learning to write: Sorne effects of network technologies", en Z. L. Berge y M. P. Coliins (eds.), Computer mediated communication and the online classromm: Higher education (vol.2), Nueva Jersey: Hampton Press.

HOFSTEDE, G. (1999): Culturas y organizaciones. El software mental, la cooperación internacional y su importancia para la supervivencia, Madrid: Alianza.

KELSEY, K. D. (2000): "Participant Interaction in a Course Delivered by Interactive Compressed Video Technologiy", en The American Journal of Distance Education, vol. 14 (1).

KERCKHOVE, N. (1999): La piel de la cultura, Barcelona: Gedisa.

LA ROSE, R., J. GREGG y M. EASTIN (1998): "Audiographie Teiecourses for the Web: An Experiment", Journal of Computer Mediated Comunication, vol. 4 (2), diciembre.

MARKUS, M. L. (1987): "Toward a 'critical mali' theory of interactive media: Universal access”, Comrnunication Research 14 (5), pp. 491-511.

MARKUS, M. L. (1990): "Toward a critical mass theory of interactive media: Universal access, interdependence and diffusion" en V. Fulk y C. Steinfeld (eds.), Organizations and communication technology, Newbury Park: Sage, pp. 194-218.

MARTIN, E. y M. Auuo (1999): "La educación a distancia en tiempos de cambios: nuevas generaciones, viejos conflictos", en E. Martín Rodríguez y M. Ahijado Quintillán (Coord.), La educación a distancia en tiempos de cambios: nuevas generaciones, viejos conflictos, Madrid: Ed. de la Torre. 
McCOMB, M. (1994): "Benefits of computer-mediated communication in college courses", en Communication Education, 43 (2), pp. 150-170.

McLUHAN, M. (1972): La galaxia Guternberg. Génesis del «horno typographycus», Madrid: Aguilar (e.o. 1962).

MOORE, M. (1989): "Editorial: Three Types of Interaction", en The American Journal of Distan- ce Education, 3 (2), pp. 1-6.

MOYA, C. (1976): «La UNED. Universidad de masas e innovación tecnológico social», en Criteríos metodológicos de la UNED, Madrid: UNED-ICE.

NORA, S, y A. Mmc (1982): La informatización de la sociedad, Madrid: Fondo de Cultura Económica (e.o. 1978).

PETERS, 0. (1983): «Distance teaching and industrial production: a comparative interpretation», en D. Stewart, D. Keegan y B. Holrnberg, Distance Education: International perspectives, Londres y Camberra: Croom Helm.

PETERS, 0. (1998): «Die Autonomie der Lernenden und ihre Bedeutung $\mathrm{Mr}$ das Fernstudium», enIAD, añoX, nl.10/il, agosto 1998, 51-82.

RAFAELI, S. (1988): "Interactivity: From new media to comunication", en R. P. Hawkins, J. M. Wiemann y S. Pingree (eds.), Advancing communication science: Merging mass and interpersonal process, Newbury Park: Sage, pp. 110-134.

RHEINGOLD, H. (1994): The virtual communify: Finding connection in a computerised world, Londres: Secker \& Warburg.

SECO, M., O. ANDRÉS Y G. RAMOS (1999): Diccionario del español actual, Madrid: Aguilar. SKINNER, B. F. (1968): The Technology of Teaching, Nueva York: Appleton, Century, Crofts.

VERTECCHI, B. (1997): "Elementi di una teoria dell'istruzione a distanza", en Istruzione a distanza, 8-9, mayo-diciembre.

VRASIDAS, CH., y M. S. MCISAAC (1999): "Factor Influencing Interaction in an Online Course", en The American Journal of Distance Education, 13 (3), pp. 22-35.

WALTHER, J.B., J.F. ANDERSON Y D.W. PAluc (1994): "Interpersonal effect in computer-mediated interaction: A meta-analysis of social and antisocial communication”, en Communication Research, n²1 (4), pp. 460-487.

\section{PERFIL ACADÉMICCO Y PROFESIONAL DEL AUTOR:}

Javier Callejo es doctor en Sociología y Licenciado en CC. de la Información (Periodismo). En los últimos años, ha realizado distintas investigaciones en los campos de la sociología de los medios de comunicación de masas y del consumo, de las que ha 
dado razón en las principales revistas especializadas. Autor del libro La audiencia activa (1995) y co-autor de diversas obras. Actualmente es Profesor Titular de Sociología en la Facultad de CC. Politicas y Sociología de la UNED y Director Adjunto del Instituto Universitario de Educación a Distancia (UNED)

\section{Javier Callejo}

Instituto Universitario de Educación a Distancia

UNED

Ciudad Universitaria

28040 Madrid (España)

Telf. 913986685

Fas. 913986693

Dirección electrónica: mcallejo@poli.uned.es

1. El anonimato también tiene sus peligros, como intervenciones exclusivamente destinadas a romper el grupo, o sus inconvenientes, como la imposibilidad de ser utilizadas para al evaluación, algo que cobra importancia en otros apartados de este trabajo.

2. Al respecto, hay que señalar la importante demanda de un mayor peso de las pruebas a distancia en el conjunto de la evaluación, por parte del alumnado. Así, según la encuesta a los alumnos del año 1997 (muestra de más de 8.000 individuos), el 33,5\% opina que habría que dar mayor importancia a las pruebas a distancia. 3 La estrecha vinculación de la frecuencia y calidad de las interacciones y la evaluación en la educación a distancia es especialmente resaltada y analizada por Bertecchi (1997). 